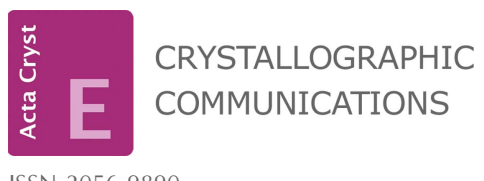

ISSN 2056-9890
Received 25 January 2019

Accepted 1 February 2019

Edited by $\mathrm{H}$. Stoeckli-Evans, University of Neuchâtel, Switzerland

Keywords: crystal structure; nickel(II); squareplanar; Schiff base; Ni...Ni interaction; Hirshfeld surfaces analysis.

CCDC reference: 1890705

Supporting information: this article has supporting information at journals.iucr.org/e

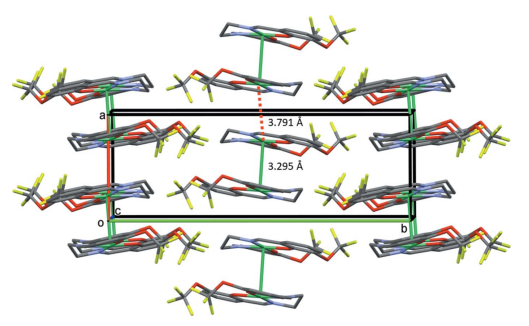

OPEN $\odot$ ACCESS

\section{Crystal structure and Hirshfeld surfaces analysis of the nickel(II) complex of the Shiff base ligand $6,6^{\prime}$-\{(1E,1'E)-[ethane-1,2-diylbis(azanylylidene)]- bis(methanylylidene)]bis[2-(trifluoromethoxy)- phenol]}

\author{
Sibel Demir Kanmazalp, ${ }^{a}$ Seher Meral, ${ }^{\mathrm{b}}$ Necmi Dege, ${ }^{\mathrm{c} *}$ Aysen Alaman Agar ${ }^{\mathrm{b}}$ and \\ Igor O. Fritsky ${ }^{d *}$

\begin{abstract}
${ }^{a}$ Gaziantep University, Technical Sciences, 27310, Gaziantep, Turkey, ${ }^{\mathbf{b}}$ Ondokuz Mayıs University, Faculty of Arts and Sciences, Department of Chemistry, 55139, Kurupelit, Samsun, Turkey, ' Ondokuz Mayıs University, Faculty of Arts and Sciences, Department of Physics, 55139, Kurupelit, Samsun, Turkey, and d Department of Chemistry, Taras Shevchenko National University of Kyiv, Volodymyrska 64/13, 01601 Kyiv, Ukraine. *Correspondence e-mail: necmid@omu.edu.tr, ifritsky@univ.kiev.ua
\end{abstract}

In the title complex, $\left(6,6^{\prime}-\left\{\left(1 E, 1^{\prime} E\right)\right.\right.$-[ethane-1,2-diylbis(azanylylidene)]bis(methanylylidene)\}bis[2-(trifluoromethoxy)phenol]- $\left.\kappa^{4} O, N, N^{\prime}, O^{\prime}\right)$ nickel(II), $\left[\mathrm{Ni}\left(\mathrm{C}_{18} \mathrm{H}_{12} \mathrm{~F}_{6} \mathrm{~N}_{2} \mathrm{O}_{4}\right)\right]$, the nickel(II) ion has a square-planar coordination sphere, being ligated by two $\mathrm{N}$ and two $\mathrm{O}$ atoms of the Schiff base ligand $6,6^{\prime}-\left\{\left(1 E, 1^{\prime} E\right)\right.$ [ethane-1,2-diylbis(azanylylidene)]bis(methanylylidene)\}bis[2-(trifluoromethoxy)phenol] (L). Inversion-related molecules are linked by a short $\mathrm{Ni} \cdot \cdots \mathrm{Ni}$ interaction of 3.2945 (6) $\AA$ forming a dimer. In the crystal, the dimers stack up the $a$ axis, with a closest $\mathrm{Ni} \cdots \mathrm{Ni}$ separation of $c a 3.791 \AA$. There are no other significant intermolecular interactions present. However, the Hirshfeld surface analysis and the two-dimensional fingerprint plots indicate that the packing is dominated by $\mathrm{H} \cdots \mathrm{F} / \mathrm{F} \cdots \mathrm{H}, \mathrm{H} \cdots \mathrm{H}, \mathrm{O} \cdots \mathrm{H} / \mathrm{H} \cdots \mathrm{O}$ and $\mathrm{C} \cdots \mathrm{H} / \mathrm{H} \cdots \mathrm{C}$ contacts.

\section{Chemical context}

Schiff bases complexes with metals are the focus of many areas of research such as the interaction of biomolecules with metals and the biological effects of metal complexes. Their $-\mathrm{OH}$ and $\mathrm{C}=\mathrm{N}$ groups are involved in the formation of covalent bonding with the metal atom; besides, these molecules are known to be easy to synthesize giving a high yield under mild conditions by solvent or solvent-free methods (Tiwari et al., 2011; Kumar et al., 2009; Kundu et al., 2009). 2-Hydroxybenzaldehyde has been used to synthesize salen-type Schiff bases, which consist of an ONNO tetradentate ligand and form five- and six-membered chelate rings with a metal atom (Atkins et al., 1985; Gupta \& Sutar, 2008). The redox character of the metal atom as well as its thermodynamic and kinetic properties results in an increase in the activity of salen-type compounds compared to organic compounds (Rijt \& Sadler, 2009). Nickel is encountered in nature as a toxic metal and therefore synthesizing compounds to selectively remove toxic materials is an important subject of research (Gupta et al., 2008). In this study, the title nickel(II) complex was synthesized from the salen-type Schiff base, $6,6^{\prime}-\left\{\left(1 E, 1^{\prime} E\right)\right.$-[ethane1,2-diylbis(azanylylidene)]bis(methanylylidene)\}bis[2-(trifluoromethoxy)phenol] (L), using nickel acetate and we report herein its crystal structure and the analysis of the Hirshfeld surface. 

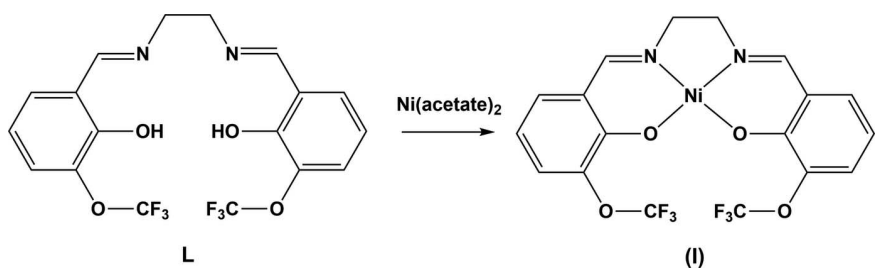

\section{Structural commentary}

The molecular structure of the asymmetric unit of the title compound (I) is shown in Fig. 1. Inversion-related complex molecules are linked by an Ni1 $\cdots \mathrm{Ni1} 1^{\mathrm{i}}$ intermetallic $d^{8} \cdots d^{8}$ interaction of 3.2945 (6) $\AA$ [Fig. 2; symmetry code (i): $-x+1$, $-y+1,-z+1]$. The nickel ion Ni1 is coordinated by two imine $\mathrm{N}$ atoms, $\mathrm{N} 6$ and $\mathrm{N} 7$, and by two phenoxo $\mathrm{O}$ atoms, $\mathrm{O} 2$ and $\mathrm{O} 3$, of the tetradentate Schiff base ligand $\mathbf{L}$. The bond lengths, $\mathrm{Ni}-\mathrm{O} 2$ and $\mathrm{Ni}-\mathrm{O} 3[1.845(2)$ and $1.840(2) \AA$, respectively], and $\mathrm{Ni}-\mathrm{N} 6$ and $\mathrm{Ni}-\mathrm{N} 7 \quad[1.839(3)$ and 1.843 (3) $\AA$, respectively] are close to the values observed for nickel complexes of similar ligands (see section Database survey). The coordinating atoms, $\mathrm{N} 6, \mathrm{~N} 7, \mathrm{O} 2, \mathrm{O} 3$, are essentially planar with no atom deviating from its mean plane by more than $0.0325 \AA$. The $\tau_{4}$ factor for four-coordinated metal atoms is $=0.04$, indicating an almost perfect square-planar coordination sphere for atom Ni1 $\left(\tau_{4}=0\right.$ for a perfect squareplanar geometry, = 1 for a perfect tetrahedral geometry; Yang et al., 2007).

\section{Supramolecular features}

In the crystal, the dimers stack up the $a$-axis direction with a $\mathrm{Ni} 1^{\mathrm{i}} \cdots \mathrm{Ni} 1^{\mathrm{ii}}$ separation of $c a .3 .791 \AA$ [see Fig. 2; symmetry codes: (i): $-x+1,-y+1,-z+1$; (ii) $x+1, y, z]$. There are no

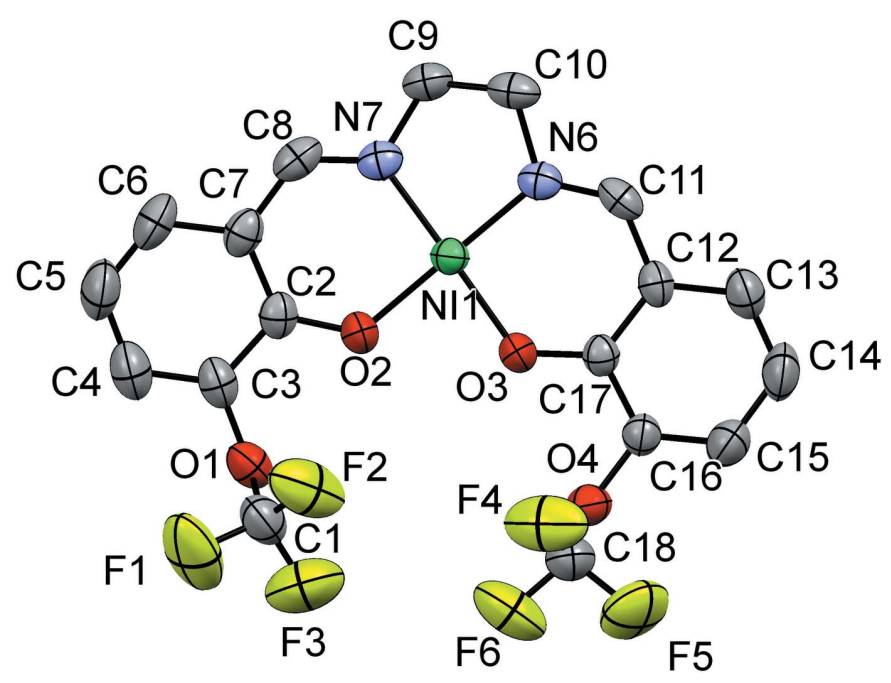

Figure 1

The molecular structure of the asymmetric unit of the title compound with the atom labelling. Displacement ellipsoids are drawn at the $50 \%$ probability level.

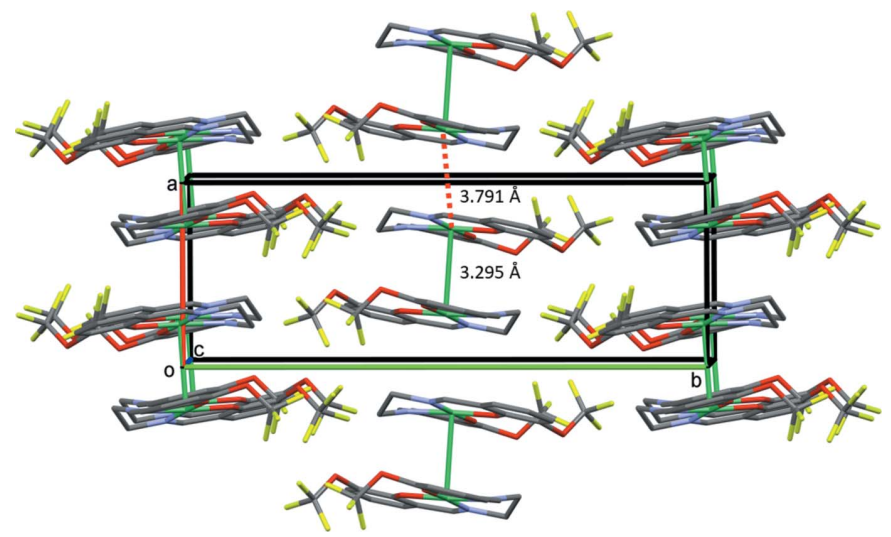

Figure 2

A view along the $c$ axis of the crystal packing of the title compound. The various $\mathrm{Ni} \cdot \cdots \mathrm{Ni}$ interactions are shown as green lines and dashed red lines. $\mathrm{H}$ atoms have been omitted for clarity.

other significant intermolecular interactions present; both $\mathrm{C}-$ $\mathrm{H} \cdots \mathrm{F}$ and $\mathrm{C}-\mathrm{H} \cdots \mathrm{O}$ interactions exceed the sum of their van der Walls radii.

\section{Database survey}

A search of the Cambridge Structural Database (CSD, Version 5.40, November 2018; Groom et al., 2016) for a 2,2'-[ethane-1,2-diylbis(iminomethylidene)]bis(phenolato)]nickel(II) moiety but with different substituents on the aromatic rings gave over 60 hits. Apart from the search skeleton (CSD refcode SAENNI), whose structure was first reported by Shkol'nikova et al. (1970), the majority of the compounds involve bis(6-methoxyphenolato) and bis(6-ethoxyphenalato) groups [see supporting information files $\mathrm{S} 1(\mathrm{H})$, $\mathrm{S} 2(\mathrm{OMe})$ and S3(OEt)]. A common feature of these complexes is the dimer formation with an $\mathrm{Ni} \cdots \mathrm{Ni}$ separation of between $c a 3.2$ to $3.9 \AA$. The same dimeric arrangement is found in the title complex, where this separation is 3.2945 (6) $\AA$. In the majority of these complexes, the $\mathrm{Ni}-$ $\mathrm{N}_{\text {imine }}$ bond lengths vary from $c a 1.837$ to $1.956 \AA$ while the $\mathrm{Ni}-\mathrm{O}_{\text {phenoxo }}$ bond lengths vary from $c a 1.834$ to $1.936 \AA$. In the title complex, the $\mathrm{Ni}-\mathrm{N}_{\text {imine }}[1.839$ (3) and 1.843 (3) $\AA$ ] and $\mathrm{Ni}-\mathrm{O}_{\text {phenoxo }}[1.840$ (2) and 1.845 (2) $\AA$ ] bond lengths fall within these limits.

\section{Hirshfeld surface analysis}

The Hirshfeld surface analysis (Spackman \& Jayatilaka, 2009) and the associated two-dimensional fingerprint plots (McKinnon et al., 2007) were performed with CrystalExplorer17 (Turner et al., 2017). Hirshfeld surfaces enable the visualization of intermolecular interactions by using different colours and colour intensity to represent short or long contacts and indicate of the relative strength of the interactions. The red regions indicate areas of close contacts shorter than the sum of van der Waals radii, while the blue and white regions represent contacts having distances greater and equal to the 


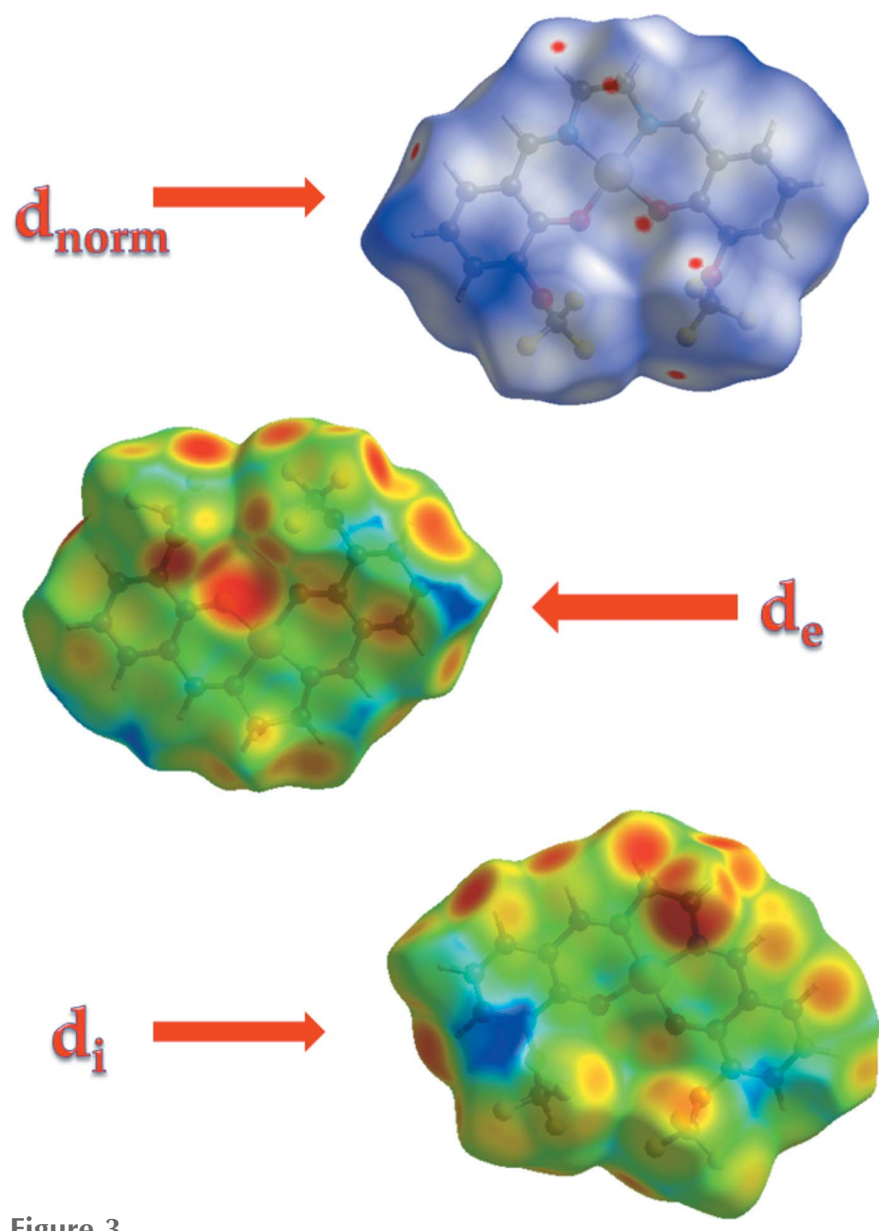

Figure 3

The Hirshfeld surface mapped over $d_{\text {norm }}, d_{\mathrm{i}}$ and $d_{\mathrm{e}}$.

sum of van der Waals radii, respectively. The three-dimensional Hirshfeld surfaces calculated for the title compound are depicted in Figs. 3 and 4. A quantitative estimate of the intermolecular interactions in the crystal structure of the title compound was obtained using Hirshfeld analysis with 2D

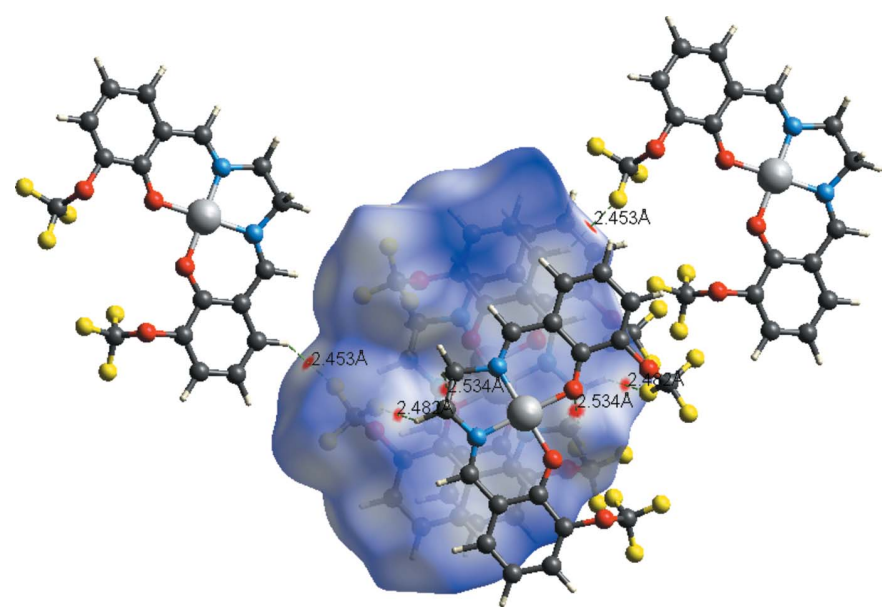

Figure 4

Hirshfeld surface mapped over $d_{\text {norm }}$, showing the weak intermolecular $\mathrm{C}-\mathrm{H} \cdots \mathrm{O}$ and $\mathrm{C}-\mathrm{H} \cdots \mathrm{F}$ contacts.
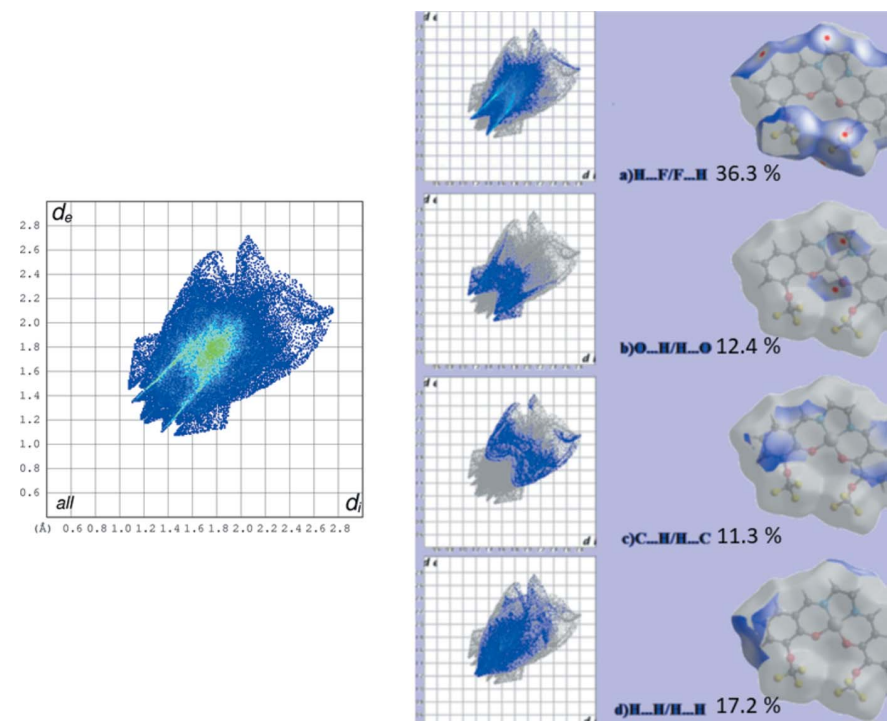

Figure 5

Total two-dimensional fingerprint plot (left) and the individual contributions to the Hirshfeld surface, together with areas of Hirshfeld surfaces involved in the intermolecular contacts (right).

fingerprint plots (Fig. 5). As can be seen from the individual fingerprint plots (Fig. 5), the most dominant contribution to the Hirshfeld surface is from $\mathrm{F} \cdots \mathrm{H} / \mathrm{H} \cdots \mathrm{F}$ interactions, with a value equal to $36.3 \%$. The scattering points spread up to $d_{\mathrm{e}}=$ $d_{\mathrm{i}}=1.4 \AA$. The other dominant forces are $\mathrm{H} \cdot \mathrm{H}(17.2 \%)$, $\mathrm{O} \cdot \mathrm{H}(12.4 \%)$ and $\mathrm{C} \cdot \mathrm{H}(11.3 \%)$ contacts. The electrostatic potential energy in the range -0.031 to 0.256 a.u., obtained using the STO-3G basis set at the Hartree-Fock level of theory, is illustrated in Fig. 6. The $\mathrm{C}-\mathrm{H} \cdots \mathrm{O}$ and $\mathrm{C}-\mathrm{H} \cdots \mathrm{F}$ donors and acceptors are shown as blue and red areas around the atoms with positive (donor) and negative (acceptors) electrostatic potentials.

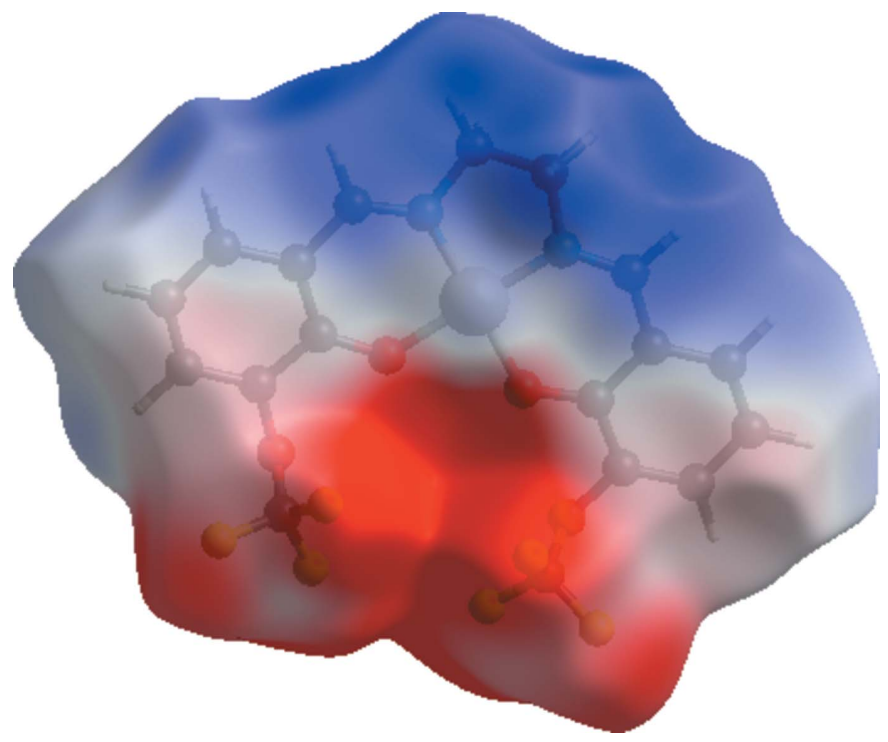

Figure 6

Electrostatic potential surface for the title compound. 


\section{Synthesis and crystallization}

The title Schiff base ligand (L), was synthesized by condensation of 2-hydroxy-3-trifluoromethoxybenzaldehyde $(0.0095 \mathrm{mmol})$ and 1,2 -ethanediamine $(0.0095 \mathrm{mmol})$ in ethanol under reflux for $c a 18 \mathrm{~h}$. The yellow product obtained was washed with ether and dried at room temperature. $\mathrm{Ni}\left(\mathrm{CH}_{3} \mathrm{COO}\right)_{2} \cdot 4 \mathrm{H}_{2} \mathrm{O}(0.0080 \mathrm{mmol})$ dissolved in $20 \mathrm{ml}$ of ethanol was added slowly to an ethanol $(20 \mathrm{ml})$ solution of $\mathbf{L}$ $(0.0080 \mathrm{mmol})$ and the mixture was refluxed for $c a 6 \mathrm{~h}$. The orange product obtained was filtered off and washed with toluene. Red rod-like crystals of the title complex were obtained by slow evaporation of a solution in ethanol at room temperature (yield 82\%, m.p. > $673 \mathrm{~K}$ ).

\section{Refinement}

Crystal data, data collection and structure refinement details are summarized in Table 1 . All $\mathrm{H}$ atoms were positioned with idealized geometry and refined as riding: $\mathrm{C}-\mathrm{H}=0.93-0.97 \AA$ with $U_{\text {iso }}(\mathrm{H})=1.2 U_{\text {eq }}(\mathrm{C})$.

\section{Funding information}

This work was supported by OMU BAP (Scientific Research Projects Unit of Ondokuz Mayıs University) under project No. PYO·FEN.1904.18.003.

\section{References}

Atkins, R., Brewer, G., Kokot, E., Mockler, G. M. \& Sinn, E. (1985). Inorg. Chem. 24, 127-134.

Farrugia, L. J. (2012). J. Appl. Cryst. 45, 849-854.

Groom, C. R., Bruno, I. J., Lightfoot, M. P. \& Ward, S. C. (2016). Acta Cryst. B72, 171-179.

Gupta, K. C. \& Sutar, A. K. (2008). Coord. Chem. Rev. 252, 14201450.

Gupta, V. K., Singh, A. K. \& Pal, M. K. (2008). Anal. Chim. Acta, 624, 223-231.

Kumar, S., Dhar, D. N. \& Saxena, P. N. (2009). J. Sci. Ind. Res. 68, 181187.

Kundu, A., Shakil, N. K., Saxena, D. B., Pankaj, Kumar, J. \& Walia, S. (2009). J. Environ. Sci. Health Part B, 44, 428-434.

Macrae, C. F., Bruno, I. J., Chisholm, J. A., Edgington, P. R., McCabe, P., Pidcock, E., Rodriguez-Monge, L., Taylor, R., van de Streek, J. \& Wood, P. A. (2008). J. Appl. Cryst. 41, 466-470.

McKinnon, J. J., Jayatilaka, D. \& Spackman, M. A. (2007). Chem. Commun. pp. 3814-3816.
Table 1

Experimental details.

\begin{tabular}{|c|c|}
\hline \multicolumn{2}{|l|}{ Crystal data } \\
\hline Chemical formula & {$\left[\mathrm{Ni}\left(\mathrm{C}_{18} \mathrm{H}_{12} \mathrm{~F}_{6} \mathrm{~N}_{2} \mathrm{O}_{4}\right)\right]$} \\
\hline$M_{\mathrm{r}}$ & 493.01 \\
\hline Crystal system, space group & Monoclinic, $P 2_{1} / n$ \\
\hline Temperature (K) & 296 \\
\hline$a, b, c(\AA)$ & $\begin{array}{l}7.0709(4), 19.8158(13) \\
13.1957(7)\end{array}$ \\
\hline$\beta\left(^{\circ}\right)$ & $99.089(4)$ \\
\hline$V\left(\AA^{3}\right)$ & $1825.71(19)$ \\
\hline$Z$ & 4 \\
\hline Radiation type & Мo $K \alpha$ \\
\hline$\mu\left(\mathrm{mm}^{-1}\right)$ & 1.15 \\
\hline Crystal size $(\mathrm{mm})$ & $0.43 \times 0.19 \times 0.05$ \\
\hline \multicolumn{2}{|l|}{ Data collection } \\
\hline Diffractometer & Stoe IPDS 2 \\
\hline Absorption correction & $\begin{array}{l}\text { Integration }(X-R E D 32 \\
\quad \text { Stoe \& Cie, 2002) }\end{array}$ \\
\hline$T_{\min }, T_{\max }$ & $0.752,0.954$ \\
\hline $\begin{array}{l}\text { No. of measured, independent and } \\
\text { observed }[I>2 \sigma(I)] \text { reflections }\end{array}$ & $10231,3594,2004$ \\
\hline$R_{\text {int }}$ & 0.069 \\
\hline$(\sin \theta / \lambda)_{\max }\left(\AA^{-1}\right)$ & 0.617 \\
\hline \multicolumn{2}{|l|}{ Refinement } \\
\hline$R\left[F^{2}>2 \sigma\left(F^{2}\right)\right], w R\left(F^{2}\right), S$ & $0.042,0.073,0.82$ \\
\hline No. of reflections & 3594 \\
\hline No. of parameters & 280 \\
\hline $\mathrm{H}$-atom treatment & $\mathrm{H}$-atom parameters constrained \\
\hline$\Delta \rho_{\max }, \Delta \rho_{\min }\left(\mathrm{e} \AA^{-3}\right)$ & $0.28,-0.26$ \\
\hline
\end{tabular}

Computer programs: $X$-AREA and X-RED32 (Stoe \& Cie, 2002), SHELXT2018 (Sheldrick, 2015a), SHELXL2018 (Sheldrick, 2015b), Mercury (Macrae et al., 2008), ORTEP-3 for Windows and WinGX (Farrugia, 2012) and PLATON (Spek, 2009).

Rijt, S. H. van \& Sadler, P. J. (2009). Drug Discov. Today, 14, 10891097.

Sheldrick, G. M. (2015a). Acta Cryst. A71, 3-8.

Sheldrick, G. M. (2015b). Acta Cryst. C71, 3-8.

Shkol'nikova, L. M., Yumal', E. M., Shugam, E. A. \& Voblikova, V. A. (1970). Zh. Strukt. Khim. (Russ. J. Struct. Chem.), 11, 886.

Spackman, M. A. \& Jayatilaka, D. (2009). CrystEngComm, 11, 19-32. Spek, A. L. (2009). Acta Cryst. D65, 148-155.

Stoe \& Cie (2002). $X$-AREA and $X$-RED32. Stoe \& Cie GmBh, Darmstadt, Germany.

Tiwari, A. D., Mishra, A. K., Mishra, S. B., Mamba, B. B., Maji, B. \& Bhattacharya, S. (2011). Spectrochim. Acta A, 79, 1050-1056.

Turner, M. J., McKinnon, J. J., Wolff, S. K., Grimwood, D. J., Spackman, P. R., Jayatilaka, D. \& Spackman, M. A. (2017). CrystalExplorer17. University of Western Australia. http://hirshfeldsurface.net

Yang, L., Powell, D. R. \& Houser, R. P. (2007). Dalton Trans. pp. 955964. 


\section{supporting information}

Acta Cryst. (2019). E75, 328-331 [https://doi.org/10.1107/S2056989019001919]

Crystal structure and Hirshfeld surfaces analysis of the nickel(II) complex of the Shiff base ligand 6,6'-\{(1E,1'E)-[ethane-1,2-diylbis(azanylylidene)]bis(methanylylidene)\}bis[2-(trifluoromethoxy)phenol]

Sibel Demir Kanmazalp, Seher Meral, Necmi Dege, Aysen Alaman Agar and Igor O. Fritsky

Computing details

Data collection: $X$-AREA (Stoe \& Cie, 2002); cell refinement: $X$-AREA (Stoe \& Cie, 2002); data reduction: X-RED32 (Stoe \& Cie, 2002); program(s) used to solve structure: SHELXT2018 (Sheldrick, 2015a); program(s) used to refine structure: SHELXL2018 (Sheldrick, 2015b); molecular graphics: ORTEP-3 for Windows (Farrugia, 2012) and Mercury (Macrae et al., 2008); software used to prepare material for publication: SHELXL2018 (Sheldrick, 2015b), WinGX (Farrugia, 2012) and PLATON (Spek, 2009).

$\left(6,6^{\prime}-\left\{\left(1 E, 1^{\prime} E\right)\right.\right.$-[Ethane-1,2-diylbis(azanylylidene)]bis(methanylylidene)\}bis[2-(trifluoromethoxy) phenol]$\left.\kappa^{4} O, N_{,} N^{\prime}, O^{\prime}\right)$ nickel(II), [Ni( $\left.\left.C_{18} \mathrm{H}_{12} \mathrm{~F}_{6} \mathrm{~N}_{2} \mathrm{O}_{4}\right)\right]$

Crystal data

$\left[\mathrm{Ni}\left(\mathrm{C}_{18} \mathrm{H}_{12} \mathrm{~F}_{6} \mathrm{~N}_{2} \mathrm{O}_{4}\right)\right]$

$M_{r}=493.01$

Monoclinic, $P 2_{1} / n$

$a=7.0709$ (4) $\AA$

$b=19.8158(13) \AA$

$c=13.1957$ (7) $\AA$

$\beta=99.089(4)^{\circ}$

$V=1825.71(19) \AA^{3}$

$Z=4$

$F(000)=992$

$D_{\mathrm{x}}=1.794 \mathrm{Mg} \mathrm{m}^{-3}$

Mo $K \alpha$ radiation, $\lambda=0.71073 \AA$

Cell parameters from 7677 reflections

$\theta=1.9-29.8^{\circ}$

$\mu=1.15 \mathrm{~mm}^{-1}$

$T=296 \mathrm{~K}$

Rod, red

$0.43 \times 0.19 \times 0.05 \mathrm{~mm}$

Data collection

Stoe IPDS 2 diffractometer

Radiation source: sealed X-ray tube, 12 x 0.4 $\mathrm{mm}$ long-fine focus

Detector resolution: 6.67 pixels $\mathrm{mm}^{-1}$ rotation method scans

Absorption correction: integration (X-RED32; Stoe \& Cie, 2002)

10231 measured reflections 3594 independent reflections 2004 reflections with $I>2 \sigma(I)$

$R_{\text {int }}=0.069$

$\theta_{\text {max }}=26.0^{\circ}, \theta_{\min }=1.9^{\circ}$

$h=-8 \rightarrow 8$

$k=-22 \rightarrow 24$

$T_{\text {min }}=0.752, T_{\max }=0.954$

$l=-16 \rightarrow 16$ 


\section{Refinement}

Refinement on $F^{2}$

Least-squares matrix: full

$R\left[F^{2}>2 \sigma\left(F^{2}\right)\right]=0.042$

$w R\left(F^{2}\right)=0.073$

$S=0.82$

3594 reflections

280 parameters

0 restraints

Primary atom site location: structure-invariant direct methods
Secondary atom site location: difference Fourier map

Hydrogen site location: inferred from neighbouring sites

$\mathrm{H}$-atom parameters constrained

$w=1 /\left[\sigma^{2}\left(F_{\mathrm{o}}^{2}\right)+(0.0208 P)^{2}\right]$ where $P=\left(F_{\mathrm{o}}^{2}+2 F_{\mathrm{c}}^{2}\right) / 3$

$(\Delta / \sigma)_{\max }<0.001$

$\Delta \rho_{\max }=0.28 \mathrm{e} \AA^{-3}$

$\Delta \rho_{\min }=-0.26$ e $\AA^{-3}$

Special details

Geometry. All esds (except the esd in the dihedral angle between two 1.s. planes) are estimated using the full covariance matrix. The cell esds are taken into account individually in the estimation of esds in distances, angles and torsion angles; correlations between esds in cell parameters are only used when they are defined by crystal symmetry. An approximate (isotropic) treatment of cell esds is used for estimating esds involving l.s. planes.

Refinement. Refinement of $\mathrm{F}^{2}$ against ALL reflections. The weighted R-factor $\mathrm{wR}$ and goodness of fit $\mathrm{S}$ are based on $\mathrm{F}^{2}$, conventional R-factors $R$ are based on $F$, with $F$ set to zero for negative $F^{2}$. The threshold expression of $\mathrm{F}^{2}>2 \operatorname{sigma}\left(\mathrm{F}^{2}\right)$ is used only for calculating R-factors(gt) etc. and is not relevant to the choice of reflections for refinement. R-factors based on $\mathrm{F}^{2}$ are statistically about twice as large as those based on F, and R- factors based on ALL data will be even larger.

Fractional atomic coordinates and isotropic or equivalent isotropic displacement parameters $\left(\AA^{2}\right)$

\begin{tabular}{|c|c|c|c|c|}
\hline & $x$ & $y$ & $z$ & $U_{\text {iso }} * / U_{\text {eq }}$ \\
\hline Nil & $0.26730(6)$ & $0.49434(2)$ & $0.49904(4)$ & $0.03711(13)$ \\
\hline $\mathrm{O} 3$ & $0.2821(4)$ & $0.40837(12)$ & $0.55236(18)$ & $0.0425(6)$ \\
\hline $\mathrm{O} 2$ & $0.3199(3)$ & $0.45369(12)$ & 0.38085 (18) & $0.0449(7)$ \\
\hline $\mathrm{O} 4$ & $0.3829(4)$ & $0.28127(12)$ & 0.60619 (19) & $0.0507(7)$ \\
\hline $\mathrm{O} 1$ & $0.4119(4)$ & $0.37488(14)$ & 0.22988 (19) & $0.0566(7)$ \\
\hline N6 & $0.2039(4)$ & $0.53396(14)$ & $0.6151(2)$ & $0.0411(8)$ \\
\hline N7 & $0.2627(4)$ & $0.58028(15)$ & $0.4452(3)$ & $0.0438(8)$ \\
\hline $\mathrm{F} 2$ & $0.1222(5)$ & $0.33925(16)$ & 0.2439 & 0.1148 (12) \\
\hline F4 & $0.1024(4)$ & $0.26758(15)$ & $0.5082(2)$ & $0.1128(12)$ \\
\hline F6 & $0.3485(5)$ & $0.21399(17)$ & $0.4791(2)$ & $0.1136(11)$ \\
\hline F5 & $0.2146(5)$ & $0.18837(15)$ & $0.6022(3)$ & $0.1122(11)$ \\
\hline $\mathrm{C} 17$ & $0.2694(5)$ & $0.39078(18)$ & 0.6458 & $0.0381(9)$ \\
\hline $\mathrm{C} 2$ & $0.3145(5)$ & $0.4817(2)$ & $0.2913(3)$ & $0.0434(10)$ \\
\hline $\mathrm{F} 1$ & $0.2352(5)$ & $0.31439(18)$ & $0.1122(2)$ & 0.1288 (14) \\
\hline $\mathrm{C} 12$ & $0.2217(5)$ & $0.4342(2)$ & 0.7222 & $0.0413(9)$ \\
\hline C11 & 0.1888 & $0.5050(2)$ & 0.7009 & $0.0443(10)$ \\
\hline H11 & 0.154060 & 0.531744 & 0.752904 & $0.053^{*}$ \\
\hline F3 & $0.3513(6)$ & $0.27078(17)$ & 0.2496 & 0.1309 (13) \\
\hline $\mathrm{C} 3$ & $0.3510(6)$ & $0.4423(2)$ & 0.2078 & $0.0501(10)$ \\
\hline $\mathrm{C} 7$ & $0.2774(5)$ & $0.5505(2)$ & 0.2689 & $0.0470(10)$ \\
\hline $\mathrm{C} 16$ & $0.3104(5)$ & 0.32354 (19) & $0.6763(3)$ & $0.0437(9)$ \\
\hline $\mathrm{C} 8$ & $0.2613(5)$ & $0.5966(2)$ & $0.3501(3)$ & $0.0496(11)$ \\
\hline H8 & 0.248688 & 0.642164 & 0.333355 & $0.060^{*}$ \\
\hline $\mathrm{C} 10$ & $0.1626(6)$ & $0.60683(18)$ & $0.6044(3)$ & $0.0524(11)$ \\
\hline H10A & 0.201695 & 0.629815 & 0.669248 & $0.063^{*}$ \\
\hline
\end{tabular}




$\begin{array}{lllll}\text { H10B } & 0.026750 & 0.614357 & 0.582588 & 0.063^{*} \\ \text { C9 } & 0.2752(6) & 0.63223(18) & 0.5249(3) & 0.0508(11) \\ \text { H9A } & 0.221970 & 0.674426 & 0.495980 & 0.061^{*} \\ \text { H9B } & 0.407695 & 0.639754 & 0.555126 & 0.061^{*} \\ \text { C13 } & 0.2102(6) & 0.4102(2) & 0.8210(3) & 0.0566(11) \\ \text { H13 } & 0.175958 & 0.439462 & 0.870112 & 0.068^{*} \\ \text { C1 } & 0.2811(8) & 0.3271(3) & 0.2096(4) & 0.0674(13) \\ \text { C18 } & 0.2617(7) & 0.2405(2) & 0.5502(4) & 0.0616(12) \\ \text { C15 } & 0.3026(6) & 0.3011(2) & 0.7727(3) & 0.0619(12) \\ \text { H15 } & 0.333337 & 0.256546 & 0.790046 & 0.074^{*} \\ \text { C6 } & 0.2706(6) & 0.5749(2) & 0.1687(4) & 0.0646(13) \\ \text { H6 } & 0.245387 & 0.620387 & 0.155453 & 0.078^{*} \\ \text { C14 } & 0.2486(7) & 0.3447(3) & 0.8456(3) & 0.0709(14) \\ \text { H14 } & 0.238953 & 0.328986 & 0.910957 & 0.085^{*} \\ \text { C4 } & 0.3449(7) & 0.4673(3) & 0.1098(3) & 0.0675(13) \\ \text { H4 } & 0.371002 & 0.439315 & 0.057199 & 0.081^{*} \\ \text { C5 } & 0.3000(7) & 0.5337(3) & 0.0908(4) & 0.0748(15) \\ \text { H5 } & 0.289649 & 0.550545 & 0.024387 & 0.090^{*}\end{array}$

Atomic displacement parameters $\left(\AA^{2}\right)$

\begin{tabular}{lllllll}
\hline & $U^{11}$ & $U^{22}$ & $U^{33}$ & $U^{12}$ & $U^{13}$ & $U^{23}$ \\
\hline Ni1 & $0.0390(2)$ & $0.0343(2)$ & $0.0376(2)$ & $0.0013(3)$ & $0.00489(16)$ & $-0.0016(3)$ \\
O3 & $0.0528(16)$ & $0.0404(14)$ & $0.0359(15)$ & $0.0000(12)$ & $0.0115(12)$ & $-0.0022(11)$ \\
O2 & $0.0556(16)$ & $0.0446(15)$ & $0.0351(15)$ & $-0.0001(12)$ & $0.0089(13)$ & $-0.0010(12)$ \\
O4 & $0.0550(17)$ & $0.0421(15)$ & $0.0554(17)$ & $0.0010(13)$ & $0.0096(14)$ & $-0.0003(13)$ \\
O1 & $0.0583(18)$ & $0.0611(19)$ & $0.0513(16)$ & $-0.0040(15)$ & $0.0117(14)$ & $-0.0148(14)$ \\
N6 & $0.0360(17)$ & $0.0402(17)$ & $0.0457(19)$ & $0.0028(14)$ & $0.0020(15)$ & $-0.0035(15)$ \\
N7 & $0.0380(18)$ & $0.0406(19)$ & $0.051(2)$ & $0.0021(14)$ & $0.0015(15)$ & $0.0006(16)$ \\
F2 & $0.094(2)$ & $0.101(2)$ & $0.168(3)$ & $-0.0372(19)$ & $0.077(2)$ & $-0.050(2)$ \\
F4 & $0.090(2)$ & $0.088(2)$ & $0.140(3)$ & $0.0169(17)$ & $-0.047(2)$ & $-0.0251(19)$ \\
F6 & $0.119(3)$ & $0.116(3)$ & $0.108(2)$ & $0.001(2)$ & $0.023(2)$ & $-0.058(2)$ \\
F5 & $0.134(3)$ & $0.068(2)$ & $0.133(3)$ & $-0.0416(19)$ & $0.017(2)$ & $0.0097(19)$ \\
C17 & $0.036(2)$ & $0.043(2)$ & $0.035(2)$ & $-0.0044(17)$ & $0.0062(17)$ & $0.0002(17)$ \\
C2 & $0.037(2)$ & $0.053(3)$ & $0.040(2)$ & $-0.0071(18)$ & $0.0055(17)$ & $0.0028(19)$ \\
F1 & $0.153(3)$ & $0.159(3)$ & $0.077(2)$ & $-0.078(3)$ & $0.025(2)$ & $-0.052(2)$ \\
C12 & $0.038(2)$ & $0.054(3)$ & $0.032(2)$ & $-0.0006(19)$ & $0.0047(17)$ & $0.0009(18)$ \\
C11 & $0.0370(19)$ & $0.054(3)$ & $0.042(2)$ & $0.003(2)$ & $0.0070(16)$ & $-0.017(2)$ \\
F3 & $0.160(3)$ & $0.065(2)$ & $0.167(3)$ & $-0.002(2)$ & $0.023(3)$ & $0.004(2)$ \\
C3 & $0.046(2)$ & $0.068(3)$ & $0.037(2)$ & $-0.007(2)$ & $0.0087(19)$ & $-0.005(2)$ \\
C7 & $0.041(2)$ & $0.052(3)$ & $0.046(2)$ & $-0.009(2)$ & $0.003(2)$ & $0.010(2)$ \\
C16 & $0.048(2)$ & $0.044(2)$ & $0.039(2)$ & $-0.0041(18)$ & $0.0069(18)$ & $0.0016(18)$ \\
C8 & $0.039(2)$ & $0.041(2)$ & $0.067(3)$ & $-0.0015(19)$ & $0.004(2)$ & $0.015(2)$ \\
C10 & $0.052(3)$ & $0.038(2)$ & $0.065(3)$ & $0.0063(18)$ & $0.002(2)$ & $-0.012(2)$ \\
C9 & $0.054(3)$ & $0.035(2)$ & $0.062(3)$ & $-0.0007(19)$ & $0.006(2)$ & $-0.0013(18)$ \\
C13 & $0.063(3)$ & $0.069(3)$ & $0.039(2)$ & $-0.008(2)$ & $0.012(2)$ & $-0.005(2)$ \\
C1 & $0.081(4)$ & $0.070(4)$ & $0.055(3)$ & $-0.011(3)$ & $0.022(3)$ & $-0.015(3)$ \\
C18 & $0.074(3)$ & $0.049(3)$ & $0.062(3)$ & $-0.001(3)$ & $0.012(3)$ & $-0.002(2)$ \\
& & & & & \\
\end{tabular}




\begin{tabular}{lllllll} 
C15 & $0.079(3)$ & $0.052(3)$ & $0.055(3)$ & $-0.004(2)$ & $0.010(2)$ & $0.010(2)$ \\
C6 & $0.068(3)$ & $0.066(3)$ & $0.059(3)$ & $-0.001(2)$ & $0.006(2)$ & $0.029(2)$ \\
C14 & $0.091(4)$ & $0.081(4)$ & $0.042(3)$ & $-0.003(3)$ & $0.015(2)$ & $0.017(3)$ \\
C4 & $0.070(3)$ & $0.091(4)$ & $0.044(3)$ & $-0.008(3)$ & $0.016(2)$ & $-0.002(2)$ \\
C5 & $0.092(4)$ & $0.088(4)$ & $0.045(3)$ & $-0.008(3)$ & $0.012(3)$ & $0.018(3)$ \\
\hline
\end{tabular}

Geometric parameters $\left(\hat{A},{ }^{\circ}\right)$

\begin{tabular}{|c|c|c|c|}
\hline Ni1-N6 & $1.839(3)$ & $\mathrm{C} 12-\mathrm{C} 11$ & $1.443(5)$ \\
\hline $\mathrm{Ni1}-\mathrm{O} 3$ & $1.840(2)$ & $\mathrm{C} 11-\mathrm{H} 11$ & 0.9300 \\
\hline $\mathrm{Ni1}-\mathrm{N} 7$ & $1.843(3)$ & $\mathrm{F} 3-\mathrm{C} 1$ & $1.299(5)$ \\
\hline $\mathrm{Ni} 1-\mathrm{O} 2$ & $1.845(2)$ & $\mathrm{C} 3-\mathrm{C} 4$ & $1.379(5)$ \\
\hline $\mathrm{O} 3-\mathrm{C} 17$ & $1.297(4)$ & $\mathrm{C} 7-\mathrm{C} 6$ & $1.401(5)$ \\
\hline $\mathrm{O} 2-\mathrm{C} 2$ & $1.301(4)$ & $\mathrm{C} 7-\mathrm{C} 8$ & $1.427(6)$ \\
\hline $\mathrm{O} 4-\mathrm{C} 18$ & $1.316(5)$ & $\mathrm{C} 16-\mathrm{C} 15$ & $1.357(5)$ \\
\hline $\mathrm{O} 4-\mathrm{C} 16$ & $1.403(4)$ & $\mathrm{C} 8-\mathrm{H} 8$ & 0.9300 \\
\hline $\mathrm{O} 1-\mathrm{C} 1$ & $1.321(5)$ & $\mathrm{C} 10-\mathrm{C} 9$ & $1.501(6)$ \\
\hline $\mathrm{O} 1-\mathrm{C} 3$ & $1.419(5)$ & $\mathrm{C} 10-\mathrm{H} 10 \mathrm{~A}$ & 0.9700 \\
\hline N6-C11 & $1.288(5)$ & $\mathrm{C} 10-\mathrm{H} 10 \mathrm{~B}$ & 0.9700 \\
\hline $\mathrm{N} 6-\mathrm{C} 10$ & $1.475(4)$ & C9-H9A & 0.9700 \\
\hline $\mathrm{N} 7-\mathrm{C} 8$ & $1.295(5)$ & $\mathrm{C} 9-\mathrm{H} 9 \mathrm{~B}$ & 0.9700 \\
\hline N7-C9 & $1.464(5)$ & $\mathrm{C} 13-\mathrm{C} 14$ & $1.354(6)$ \\
\hline $\mathrm{F} 2-\mathrm{C} 1$ & $1.298(5)$ & $\mathrm{C} 13-\mathrm{H} 13$ & 0.9300 \\
\hline $\mathrm{F} 4-\mathrm{C} 18$ & $1.291(5)$ & $\mathrm{C} 15-\mathrm{C} 14$ & $1.390(6)$ \\
\hline $\mathrm{F} 6-\mathrm{C} 18$ & $1.311(5)$ & $\mathrm{C} 15-\mathrm{H} 15$ & 0.9300 \\
\hline $\mathrm{F} 5-\mathrm{C} 18$ & $1.313(5)$ & $\mathrm{C} 6-\mathrm{C} 5$ & $1.355(6)$ \\
\hline $\mathrm{C} 17-\mathrm{C} 12$ & $1.407(5)$ & $\mathrm{C} 6-\mathrm{H} 6$ & 0.9300 \\
\hline $\mathrm{C} 17-\mathrm{C} 16$ & $1.409(5)$ & $\mathrm{C} 14-\mathrm{H} 14$ & 0.9300 \\
\hline $\mathrm{C} 2-\mathrm{C} 3$ & $1.409(5)$ & $\mathrm{C} 4-\mathrm{C} 5$ & $1.367(6)$ \\
\hline $\mathrm{C} 2-\mathrm{C} 7$ & $1.410(5)$ & $\mathrm{C} 4-\mathrm{H} 4$ & 0.9300 \\
\hline $\mathrm{F} 1-\mathrm{C} 1$ & $1.300(5)$ & $\mathrm{C} 5-\mathrm{H} 5$ & 0.9300 \\
\hline $\mathrm{C} 12-\mathrm{C} 13$ & $1.402(5)$ & & \\
\hline $\mathrm{N} 6-\mathrm{Ni1}-\mathrm{O} 3$ & $94.78(12)$ & $\mathrm{N} 6-\mathrm{C} 10-\mathrm{H} 10 \mathrm{~A}$ & 110.5 \\
\hline N6-Ni1-N7 & $86.27(14)$ & $\mathrm{C} 9-\mathrm{C} 10-\mathrm{H} 10 \mathrm{~A}$ & 110.5 \\
\hline $\mathrm{O} 3-\mathrm{Ni1}-\mathrm{N} 7$ & $177.70(14)$ & $\mathrm{N} 6-\mathrm{C} 10-\mathrm{H} 10 \mathrm{~B}$ & 110.5 \\
\hline $\mathrm{N} 6-\mathrm{Ni1}-\mathrm{O} 2$ & $177.53(13)$ & $\mathrm{C} 9-\mathrm{C} 10-\mathrm{H} 10 \mathrm{~B}$ & 110.5 \\
\hline $\mathrm{O} 3-\mathrm{Ni1}-\mathrm{O} 2$ & $84.95(11)$ & $\mathrm{H} 10 \mathrm{~A}-\mathrm{C} 10-\mathrm{H} 10 \mathrm{~B}$ & 108.7 \\
\hline $\mathrm{N} 7-\mathrm{Ni1}-\mathrm{O} 2$ & $94.08(14)$ & $\mathrm{N} 7-\mathrm{C} 9-\mathrm{C} 10$ & $106.7(3)$ \\
\hline $\mathrm{C} 17-\mathrm{O} 3-\mathrm{Ni1}$ & $127.1(2)$ & $\mathrm{N} 7-\mathrm{C} 9-\mathrm{H} 9 \mathrm{~A}$ & 110.4 \\
\hline $\mathrm{C} 2-\mathrm{O} 2-\mathrm{Ni} 1$ & $126.9(2)$ & $\mathrm{C} 10-\mathrm{C} 9-\mathrm{H} 9 \mathrm{~A}$ & 110.4 \\
\hline $\mathrm{C} 18-\mathrm{O} 4-\mathrm{C} 16$ & $117.5(3)$ & N7-C9-H9B & 110.4 \\
\hline $\mathrm{C} 1-\mathrm{O} 1-\mathrm{C} 3$ & $116.9(3)$ & $\mathrm{C} 10-\mathrm{C} 9-\mathrm{H} 9 \mathrm{~B}$ & 110.4 \\
\hline $\mathrm{C} 11-\mathrm{N} 6-\mathrm{C} 10$ & $118.5(4)$ & $\mathrm{H} 9 \mathrm{~A}-\mathrm{C} 9-\mathrm{H} 9 \mathrm{~B}$ & 108.6 \\
\hline $\mathrm{C} 11-\mathrm{N} 6-\mathrm{Ni1}$ & $127.4(3)$ & $\mathrm{C} 14-\mathrm{C} 13-\mathrm{C} 12$ & $120.6(4)$ \\
\hline $\mathrm{C} 10-\mathrm{N} 6-\mathrm{Ni1}$ & $114.1(3)$ & $\mathrm{C} 14-\mathrm{C} 13-\mathrm{H} 13$ & 119.7 \\
\hline $\mathrm{C} 8-\mathrm{N} 7-\mathrm{C} 9$ & $120.7(3)$ & $\mathrm{C} 12-\mathrm{C} 13-\mathrm{H} 13$ & 119.7 \\
\hline $\mathrm{C} 8-\mathrm{N} 7-\mathrm{Ni} 1$ & $127.0(3)$ & $\mathrm{F} 2-\mathrm{C} 1-\mathrm{F} 3$ & $108.3(5)$ \\
\hline
\end{tabular}




\begin{tabular}{|c|c|c|c|}
\hline $\mathrm{C} 9-\mathrm{N} 7-\mathrm{Ni1}$ & $112.2(2)$ & $\mathrm{F} 2-\mathrm{C} 1-\mathrm{F} 1$ & $106.6(4)$ \\
\hline $\mathrm{O} 3-\mathrm{C} 17-\mathrm{C} 12$ & $125.3(3)$ & $\mathrm{F} 3-\mathrm{C} 1-\mathrm{F} 1$ & $104.7(4)$ \\
\hline $\mathrm{O} 3-\mathrm{C} 17-\mathrm{C} 16$ & $118.9(3)$ & $\mathrm{F} 2-\mathrm{C} 1-\mathrm{O} 1$ & $114.4(4)$ \\
\hline $\mathrm{C} 12-\mathrm{C} 17-\mathrm{C} 16$ & $115.8(3)$ & $\mathrm{F} 3-\mathrm{C} 1-\mathrm{O} 1$ & $108.8(4)$ \\
\hline $\mathrm{O} 2-\mathrm{C} 2-\mathrm{C} 3$ & $119.4(4)$ & $\mathrm{F} 1-\mathrm{C} 1-\mathrm{O} 1$ & $113.4(4)$ \\
\hline $\mathrm{O} 2-\mathrm{C} 2-\mathrm{C} 7$ & $125.6(4)$ & $\mathrm{F} 4-\mathrm{C} 18-\mathrm{F} 6$ & $109.6(4)$ \\
\hline $\mathrm{C} 3-\mathrm{C} 2-\mathrm{C} 7$ & $115.0(4)$ & $\mathrm{F} 4-\mathrm{C} 18-\mathrm{F} 5$ & $106.0(4)$ \\
\hline $\mathrm{C} 13-\mathrm{C} 12-\mathrm{C} 17$ & $121.0(4)$ & $\mathrm{F} 6-\mathrm{C} 18-\mathrm{F} 5$ & $104.2(4)$ \\
\hline $\mathrm{C} 13-\mathrm{C} 12-\mathrm{C} 11$ & $118.7(4)$ & $\mathrm{F} 4-\mathrm{C} 18-\mathrm{O} 4$ & $115.7(4)$ \\
\hline $\mathrm{C} 17-\mathrm{C} 12-\mathrm{C} 11$ & $120.3(3)$ & $\mathrm{F} 6-\mathrm{C} 18-\mathrm{O} 4$ & $108.1(4)$ \\
\hline $\mathrm{N} 6-\mathrm{C} 11-\mathrm{C} 12$ & $124.8(4)$ & $\mathrm{F} 5-\mathrm{C} 18-\mathrm{O} 4$ & $112.7(4)$ \\
\hline N6-C11-H11 & 117.6 & $\mathrm{C} 16-\mathrm{C} 15-\mathrm{C} 14$ & $120.1(4)$ \\
\hline $\mathrm{C} 12-\mathrm{C} 11-\mathrm{H} 11$ & 117.6 & $\mathrm{C} 16-\mathrm{C} 15-\mathrm{H} 15$ & 119.9 \\
\hline $\mathrm{C} 4-\mathrm{C} 3-\mathrm{C} 2$ & $123.5(4)$ & $\mathrm{C} 14-\mathrm{C} 15-\mathrm{H} 15$ & 119.9 \\
\hline $\mathrm{C} 4-\mathrm{C} 3-\mathrm{O} 1$ & $119.7(4)$ & $\mathrm{C} 5-\mathrm{C} 6-\mathrm{C} 7$ & $121.4(4)$ \\
\hline $\mathrm{C} 2-\mathrm{C} 3-\mathrm{O} 1$ & $116.7(3)$ & $\mathrm{C} 5-\mathrm{C} 6-\mathrm{H} 6$ & 119.3 \\
\hline $\mathrm{C} 6-\mathrm{C} 7-\mathrm{C} 2$ & $120.6(4)$ & $\mathrm{C} 7-\mathrm{C} 6-\mathrm{H} 6$ & 119.3 \\
\hline $\mathrm{C} 6-\mathrm{C} 7-\mathrm{C} 8$ & $119.5(4)$ & $\mathrm{C} 13-\mathrm{C} 14-\mathrm{C} 15$ & $119.7(4)$ \\
\hline $\mathrm{C} 2-\mathrm{C} 7-\mathrm{C} 8$ & $119.7(4)$ & $\mathrm{C} 13-\mathrm{C} 14-\mathrm{H} 14$ & 120.1 \\
\hline $\mathrm{C} 15-\mathrm{C} 16-\mathrm{O} 4$ & $119.7(4)$ & $\mathrm{C} 15-\mathrm{C} 14-\mathrm{H} 14$ & 120.1 \\
\hline $\mathrm{C} 15-\mathrm{C} 16-\mathrm{C} 17$ & $122.7(4)$ & $\mathrm{C} 5-\mathrm{C} 4-\mathrm{C} 3$ & $119.4(5)$ \\
\hline $\mathrm{O} 4-\mathrm{C} 16-\mathrm{C} 17$ & $117.2(3)$ & $\mathrm{C} 5-\mathrm{C} 4-\mathrm{H} 4$ & 120.3 \\
\hline $\mathrm{N} 7-\mathrm{C} 8-\mathrm{C} 7$ & $125.4(4)$ & $\mathrm{C} 3-\mathrm{C} 4-\mathrm{H} 4$ & 120.3 \\
\hline $\mathrm{N} 7-\mathrm{C} 8-\mathrm{H} 8$ & 117.3 & $\mathrm{C} 6-\mathrm{C} 5-\mathrm{C} 4$ & $120.0(5)$ \\
\hline $\mathrm{C} 7-\mathrm{C} 8-\mathrm{H} 8$ & 117.3 & $\mathrm{C} 6-\mathrm{C} 5-\mathrm{H} 5$ & 120.0 \\
\hline $\mathrm{N} 6-\mathrm{C} 10-\mathrm{C} 9$ & $106.1(3)$ & $\mathrm{C} 4-\mathrm{C} 5-\mathrm{H} 5$ & 120.0 \\
\hline $\mathrm{N} 6-\mathrm{Ni1}-\mathrm{O} 3-\mathrm{C} 17$ & $-6.3(3)$ & $\mathrm{C} 18-\mathrm{O} 4-\mathrm{C} 16-\mathrm{C} 15$ & $88.3(4)$ \\
\hline $\mathrm{O} 2-\mathrm{Ni} 1-\mathrm{O} 3-\mathrm{C} 17$ & $176.1(3)$ & $\mathrm{C} 18-\mathrm{O} 4-\mathrm{C} 16-\mathrm{C} 17$ & $-98.9(4)$ \\
\hline $\mathrm{O} 3-\mathrm{Ni} 1-\mathrm{O} 2-\mathrm{C} 2$ & $171.3(3)$ & $\mathrm{O} 3-\mathrm{C} 17-\mathrm{C} 16-\mathrm{C} 15$ & $179.3(4)$ \\
\hline $\mathrm{N} 7-\mathrm{Ni1}-\mathrm{O} 2-\mathrm{C} 2$ & $-10.8(3)$ & $\mathrm{C} 12-\mathrm{C} 17-\mathrm{C} 16-\mathrm{C} 15$ & $0.8(6)$ \\
\hline $\mathrm{O} 3-\mathrm{Ni1}-\mathrm{N} 6-\mathrm{C} 11$ & $2.6(3)$ & $\mathrm{O} 3-\mathrm{C} 17-\mathrm{C} 16-\mathrm{O} 4$ & $6.7(5)$ \\
\hline $\mathrm{N} 7-\mathrm{Ni1}-\mathrm{N} 6-\mathrm{C} 11$ & $-175.3(3)$ & $\mathrm{C} 12-\mathrm{C} 17-\mathrm{C} 16-\mathrm{O} 4$ & $-171.8(3)$ \\
\hline $\mathrm{O} 3-\mathrm{Ni1}-\mathrm{N} 6-\mathrm{C} 10$ & $-175.9(2)$ & $\mathrm{C} 9-\mathrm{N} 7-\mathrm{C} 8-\mathrm{C} 7$ & $170.4(3)$ \\
\hline $\mathrm{N} 7-\mathrm{Ni1}-\mathrm{N} 6-\mathrm{C} 10$ & $6.2(2)$ & $\mathrm{Ni1}-\mathrm{N} 7-\mathrm{C} 8-\mathrm{C} 7$ & $-5.0(6)$ \\
\hline $\mathrm{N} 6-\mathrm{Ni1}-\mathrm{N} 7-\mathrm{C} 8$ & $-166.3(3)$ & $\mathrm{C} 6-\mathrm{C} 7-\mathrm{C} 8-\mathrm{N} 7$ & $179.0(4)$ \\
\hline $\mathrm{O} 2-\mathrm{Ni} 1-\mathrm{N} 7-\mathrm{C} 8$ & $11.3(3)$ & $\mathrm{C} 2-\mathrm{C} 7-\mathrm{C} 8-\mathrm{N} 7$ & $-5.8(6)$ \\
\hline $\mathrm{N} 6-\mathrm{Ni} 1-\mathrm{N} 7-\mathrm{C} 9$ & $18.0(2)$ & $\mathrm{C} 11-\mathrm{N} 6-\mathrm{C} 10-\mathrm{C} 9$ & $153.9(3)$ \\
\hline $\mathrm{O} 2-\mathrm{Ni1}-\mathrm{N} 7-\mathrm{C} 9$ & $-164.5(2)$ & $\mathrm{Ni} 1-\mathrm{N} 6-\mathrm{C} 10-\mathrm{C} 9$ & $-27.4(3)$ \\
\hline $\mathrm{Ni} 1-\mathrm{O} 3-\mathrm{C} 17-\mathrm{C} 12$ & $7.0(5)$ & $\mathrm{C} 8-\mathrm{N} 7-\mathrm{C} 9-\mathrm{C} 10$ & $147.0(3)$ \\
\hline $\mathrm{Ni1}-\mathrm{O} 3-\mathrm{C} 17-\mathrm{C} 16$ & $-171.4(2)$ & $\mathrm{Ni} 1-\mathrm{N} 7-\mathrm{C} 9-\mathrm{C} 10$ & $-36.9(3)$ \\
\hline $\mathrm{Ni} 1-\mathrm{O} 2-\mathrm{C} 2-\mathrm{C} 3$ & $-177.2(3)$ & $\mathrm{N} 6-\mathrm{C} 10-\mathrm{C} 9-\mathrm{N} 7$ & $39.5(4)$ \\
\hline $\mathrm{Ni1}-\mathrm{O} 2-\mathrm{C} 2-\mathrm{C} 7$ & $3.9(5)$ & $\mathrm{C} 17-\mathrm{C} 12-\mathrm{C} 13-\mathrm{C} 14$ & $1.3(6)$ \\
\hline $\mathrm{O} 3-\mathrm{C} 17-\mathrm{C} 12-\mathrm{C} 13$ & $179.5(3)$ & $\mathrm{C} 11-\mathrm{C} 12-\mathrm{C} 13-\mathrm{C} 14$ & $-177.0(4)$ \\
\hline $\mathrm{C} 16-\mathrm{C} 17-\mathrm{C} 12-\mathrm{C} 13$ & $-2.1(5)$ & $\mathrm{C} 3-\mathrm{O} 1-\mathrm{C} 1-\mathrm{F} 2$ & $-48.2(6)$ \\
\hline $\mathrm{O} 3-\mathrm{C} 17-\mathrm{C} 12-\mathrm{C} 11$ & $-2.2(6)$ & $\mathrm{C} 3-\mathrm{O} 1-\mathrm{C} 1-\mathrm{F} 3$ & $-169.5(4)$ \\
\hline $\mathrm{C} 16-\mathrm{C} 17-\mathrm{C} 12-\mathrm{C} 11$ & $176.1(3)$ & $\mathrm{C} 3-\mathrm{O} 1-\mathrm{C} 1-\mathrm{F} 1$ & $74.4(6)$ \\
\hline $\mathrm{C} 10-\mathrm{N} 6-\mathrm{C} 11-\mathrm{C} 12$ & $179.1(3)$ & $\mathrm{C} 16-\mathrm{O} 4-\mathrm{C} 18-\mathrm{F} 4$ & $46.5(6)$ \\
\hline
\end{tabular}




$\begin{array}{llll}\mathrm{Ni} 1-\mathrm{N} 6-\mathrm{C} 11-\mathrm{C} 12 & 0.6(5) & \mathrm{C} 16-\mathrm{O} 4-\mathrm{C} 18-\mathrm{F} 6 & 169.7(3) \\ \mathrm{C} 13-\mathrm{C} 12-\mathrm{C} 11-\mathrm{N} 6 & 176.5(3) & \mathrm{C} 16-\mathrm{O} 4-\mathrm{C} 18-\mathrm{F} 5 & -75.7(5) \\ \mathrm{C} 17-\mathrm{C} 12-\mathrm{C} 11-\mathrm{N} 6 & -1.7(5) & \mathrm{O} 4-\mathrm{C} 16-\mathrm{C} 15-\mathrm{C} 14 & 173.7(4) \\ \mathrm{O} 2-\mathrm{C} 2-\mathrm{C} 3-\mathrm{C} 4 & 178.7(4) & \mathrm{C} 17-\mathrm{C} 16-\mathrm{C} 15-\mathrm{C} 14 & 1.4(6) \\ \mathrm{C} 7-\mathrm{C} 2-\mathrm{C} 3-\mathrm{C} 4 & -2.3(6) & \mathrm{C} 2-\mathrm{C} 7-\mathrm{C} 6-\mathrm{C} 5 & -0.3(7) \\ \mathrm{O} 2-\mathrm{C} 2-\mathrm{C} 3-\mathrm{O} 1 & -6.5(5) & \mathrm{C} 8-\mathrm{C} 7-\mathrm{C} 6-\mathrm{C} 5 & 174.9(4) \\ \mathrm{C} 7-\mathrm{C} 2-\mathrm{C} 3-\mathrm{O} 1 & 172.5(3) & \mathrm{C} 12-\mathrm{C} 13-\mathrm{C} 14-\mathrm{C} 15 & 0.9(7) \\ \mathrm{C} 1-\mathrm{O} 1-\mathrm{C} 3-\mathrm{C} 4 & -84.6(5) & \mathrm{C} 16-\mathrm{C} 15-\mathrm{C} 14-\mathrm{C} 13 & -2.2(7) \\ \mathrm{C} 1-\mathrm{O} 1-\mathrm{C} 3-\mathrm{C} 2 & 100.4(4) & \mathrm{C} 2-\mathrm{C} 3-\mathrm{C} 4-\mathrm{C} 5 & -0.5(7) \\ \mathrm{O} 2-\mathrm{C} 2-\mathrm{C} 7-\mathrm{C} 6 & -178.5(4) & \mathrm{O} 1-\mathrm{C} 3-\mathrm{C} 4-\mathrm{C} 5 & -175.1(4) \\ \mathrm{C} 3-\mathrm{C} 2-\mathrm{C} 7-\mathrm{C} 6 & 2.6(6) & \mathrm{C} 7-\mathrm{C} 6-\mathrm{C} 5-\mathrm{C} 4 & -2.6(8) \\ \mathrm{O} 2-\mathrm{C} 2-\mathrm{C} 7-\mathrm{C} 8 & 6.4(6) & \mathrm{C} 3-\mathrm{C} 4-\mathrm{C} 5-\mathrm{C} 6 & 3.0(8) \\ \mathrm{C} 3-\mathrm{C} 2-\mathrm{C} 7-\mathrm{C} 8 & -172.5(3) & & \end{array}$

Hydrogen-bond geometry $\left(A,{ }^{\circ}\right)$

\begin{tabular}{lllll}
\hline$D-\mathrm{H} \cdots A$ & $D-\mathrm{H}$ & $\mathrm{H} \cdots A$ & $D \cdots A$ & $D-\mathrm{H} \cdots A$ \\
\hline $\mathrm{C} 10-\mathrm{H} 10 B \cdots \mathrm{O} 3^{\mathrm{i}}$ & 0.97 & 2.63 & $3.494(4)$ & 149 \\
$\mathrm{C} 9-\mathrm{H} 9 A \cdots \mathrm{F} 4^{\mathrm{i}}$ & 0.97 & 2.56 & $3.301(5)$ & 133 \\
$\mathrm{C} 6-\mathrm{H} 6 \cdots \mathrm{F} 66^{\mathrm{ii}}$ & 0.93 & 2.58 & $3.405(5)$ & 148 \\
\hline
\end{tabular}

Symmetry codes: (i) $-x,-y+1,-z+1$; (ii) $-x+1 / 2, y+1 / 2,-z+1 / 2$. 